\title{
Results from Super-Kamiokande
}

Y. Takeuchi for the Super-Kamiokande collaboration

Dept. of Physics, Graduate School of Science, Kobe University, 1-1 Rokkodai-cho, Nada, Kobe 657-8501

\begin{abstract}
The recent results from Super-Kamiokande (SK) are reported. On atmospheric neutrino analysis, we have performed a full 3-flavor oscillation analysis with SK-I+II+III data. A CPT violation study on atmospheric neutrino is also done with SK-I+II+III data. On solar neutrino analysis, a 3-flavor oscillation analysis with SK-III data is performed.
\end{abstract}

Keywords: Solar neutrino, Atmospheric neutrino

\section{Super-Kamiokande detector}

Super-Kamiokande (SK) [1] is a cylindrical water Cherenkov detector with 50-kton of purified water and about 11000 of 20-inch PMT's. It is located $1000 \mathrm{~m}$ underground (2700 m water equivalence) in Kamioka Observatory, ICRR, University of Tokyo in Mozumi mine in Gifu prefecture in Japan. Figure 1 shows the inside of the SK detector. The fiducial volume of the SK detector is $22.5 \mathrm{kton}$. The main observation targets of SK detector are solar neutrinos, atmospheric neutrinos, supernova neutrinos, and nucleon decays. SK is the largest detector in the world for these physics targets.

SK has started its observation in 1996. Table 1 shows a summary of the SK experiments. Currently, SK-IV is running with the lowest energy threshold at the electron total energy, $E_{\text {total }}=4.5 \mathrm{MeV}$. In SK-IV, we have replaced the entire front-end electronics system. All the hit information of PMT's can be read by the electronics system [2]. In SK-IV, we can apply appropriate event time windows, for example, $1.5 \mu$ s for the lowenergy (= high rate) events, $40 \mu$ s for the normal events, $540 \mu \mathrm{s}$ for the high-energy without OD activity events, and $\pm 512 \mu$ s around the beam spill timing of the T2K

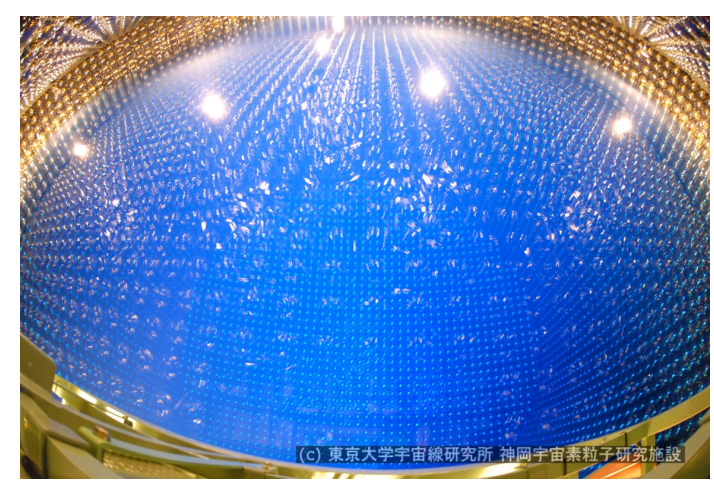

Figure 1: Super-Kamiokande detector during water filling in June 2006.

Email address: takeuchi@phys.sci . kobe-u . ac.jp (Y. Takeuchi for the Super-Kamiokande collaboration) 


\begin{tabular}{llrrc}
\hline \hline Phase & Run period & Number of ID PMT & $\begin{array}{r}\text { Photo coverage } \\
(\%)\end{array}$ & $\begin{array}{r}\text { Analysis energy threshold } \\
\text { Total / Kinetic Energy }\end{array}$ \\
\hline SK-I & Apr. 1996 - Jul. 2001 & 11146 & 40 & $5.0 \mathrm{MeV} / 4.5 \mathrm{MeV}$ \\
SK-II & Dec. 2002 - Oct. 2005 & 5182 & 19 & $7.0 \mathrm{MeV} / 6.5 \mathrm{MeV}$ \\
SK-III & Jul. 2006 - Aug. 2008 & 11129 & 40 & $5.0 \mathrm{MeV} / 4.5 \mathrm{MeV}$ \\
SK-IV & Sep. 2008 - today & 11129 & 40 & $(4.5 \mathrm{MeV} / 4.0 \mathrm{MeV})$ \\
\hline \hline
\end{tabular}

Table 1: Summary of the experimental phases in SK.

experiment. In future, we would like to lower the energy threshold down to $E_{\text {total }}=4.0 \mathrm{MeV}$ or less.

\section{Atmospheric neutrino results}

Cosmic-ray interactions in the atmosphere produce neutrinos. They are called atmospheric neutrinos. In the SK detector, atmospheric neutrino events are categorized into fully contained (FC) events, partially contained (PC) events, and upward going muons.

FC events deposit all of their Cherenkov light in the inner detector (ID) in SK, while PC events include punch-through tracks which deposit some Cherenkov light in the outer detector (OD). The neutrino interaction vertex is required to be reconstructed within the 22.5 kton fiducial volume. The FC events are classified into "sub-GeV" (visible energy, $E_{v i s}<1330 \mathrm{MeV}$ ) and "multi-GeV" $\left(E_{v i s}>1330 \mathrm{MeV}\right)$. These events are further separated into sub-samples based on the number of observed Cherenkov rings. Single- and multi-ring are then divided into electron-like (e-like) or muon-like ( $\mu$ like) samples depending on pattern identification of the most energetic Cherenkov ring. The sub-GeV samples are additionally divided based on their number of decayelectrons and their likelihood of being a $\pi^{0}$.

The PC events are separated into "OD stopping" and "OD through-going" categories based on the amount of light deposit by the exiting particle in the OD.

Energetic atmospheric muon neutrinos passing through the Earth interact with rock surrounding the detector, and produce muons via charged current interactions. These neutrino events are observed as upward going muons. Upward going muons are classified into two types. One is "upward through-going muons" which have passed through the detector, and the other is "upward stopping muons" which come into and stop inside the detector. The upward through-going muons are subdivided into "showering" and "non-showering" based on whether their Cherenkov pattern is consistent with light emitted from an electromagnetic shower produced by a very high energy muon.
Table 2: Atmospheric neutrino livetime and the number of observed FC and PC events for each SK phase.

\begin{tabular}{crrr}
\hline & Livetime (days) & FC & PC \\
\hline SK-I & 1,489 & 12,232 & 896 \\
SK-II & 799 & 6,584 & 429 \\
SK-III & 518 & 4,356 & 343 \\
\hline
\end{tabular}

Table 3: Atmospheric neutrino induced upward-going muon livetime and the number of observed events for each SK phase.

\begin{tabular}{crrr}
\hline & Livetime (days) & through-going & stopping \\
\hline SK-I & 1,646 & 1,856 & 458 \\
SK-II & 828 & 889 & 228 \\
SK-III & 636 & 735 & 210 \\
\hline
\end{tabular}

The livetime and number of observed events for the first three SK phases are summarized in Table 2 and 3 . respectively.

The zenith angle and lepton momentum distributions for each of the above samples compared with the atmospheric neutrino Monte Carlo (MC) predictions are shown in Figure 2

In June 2009, we have summarized a preliminary results of the neutrino oscillation analysis using SK-I, II and III atmospheric neutrino data set. Red contours in Figure 3 show the allowed neutrino oscillation parameter regions for $v_{\mu} \leftrightarrow v_{\tau}$ oscillations. The allowed oscillation parameter region from the zenith angle analysis is $\sin ^{2} 2 \theta_{23}>0.96(90 \%$ C.L. $)$ and $\Delta m_{23}^{2}=(2.11+0.11-$ $0.19) \times 10^{-3} \mathrm{eV}^{2}$. The allowed oscillation parameter region from the $\mathrm{L} / \mathrm{E}$ analysis is $\sin ^{2} 2 \theta_{23}>0.96(90 \%$ C.L.) and $\Delta m_{23}^{2}=(2.19+0.14-0.13) \times 10^{-3} \mathrm{eV}^{2}$.

In May 2010, we have performed a full 3-flavor oscillation analysis considering all the mixing parameters and the $\mathrm{CP}$ violating term. The matter effect in the Earth is also considered in this calculation and both the normal and inverted mass hierarchies are tested. Figure 4 shows the allowed regions for $\left(\Delta m_{23}^{2}, \sin ^{2} 2 \theta_{23}\right),\left(\Delta m_{23}^{2}\right.$, $\left.\sin ^{2} \theta_{13}\right)$, and $\left(\sin ^{2} \theta_{13}, \delta_{c p}\right)$ for the normal and inverted mass hierarchies with SK-I+II+III data. A numerical 


\begin{tabular}{ccccc} 
& Parameter & Best point & $90 \%$ C.L. allowed & $68 \%$ C.L. allowed \\
\cline { 2 - 5 } Normal hierarchy $\left(\chi_{\min }^{2}=469.94 / 416\right.$ dof $)$ & $\Delta m_{23}^{2}\left(\times 10^{3}\right)$ & $2.11 \mathrm{eV}^{2}$ & $1.88-2.75 \mathrm{eV}^{2}$ & $1.99-2.54 \mathrm{eV}^{2}$ \\
& $\sin ^{2} \theta_{23}$ & 0.525 & $0.406-0.629$ & $0.441-0.597$ \\
& $\sin ^{2} \theta_{13}$ & 0.006 & $<0.066$ & $<0.036$ \\
& $\mathrm{CP}-\delta$ & $220^{\circ}$ & - & $140.8^{\circ}-297.3^{\circ}$ \\
\cline { 2 - 5 } & Parameter & Best point & $90 \%$ C.L. allowed & $68 \%$ C.L. allowed \\
\cline { 2 - 5 } Inverted hierarchy $\left(\chi_{\min }^{2}=468.34 / 416\right.$ dof) & $\Delta m_{23}^{2}\left(\times 10^{3}\right)$ & $2.51 \mathrm{eV}^{2}$ & $1.98-2.81 \mathrm{eV}^{2}$ & $2.09-2.64 \mathrm{eV}^{2}$ \\
& $\sin ^{2} \theta_{23}$ & 0.575 & $0.426-0.644$ & $0.501-0.623$ \\
& $\sin ^{2} \theta_{13}$ & 0.044 & $<0.122$ & $0.0122-0.0850$ \\
& $\mathrm{CP}-\delta$ & $220^{\circ}$ & $121.4^{\circ}-319.1^{\circ}$ & $165.6^{\circ}-280.4^{\circ}$ \\
\hline \hline
\end{tabular}

Table 4: Summary of the full 3-flavor oscillation analysis results in SK-I+II+III (preliminary). The $\sin ^{2} \theta_{12}$ and $\Delta m_{12}^{2}$ are fixed at 0.304 and $7.66 \times 10^{-5} \mathrm{eV}^{2}$, respectively.
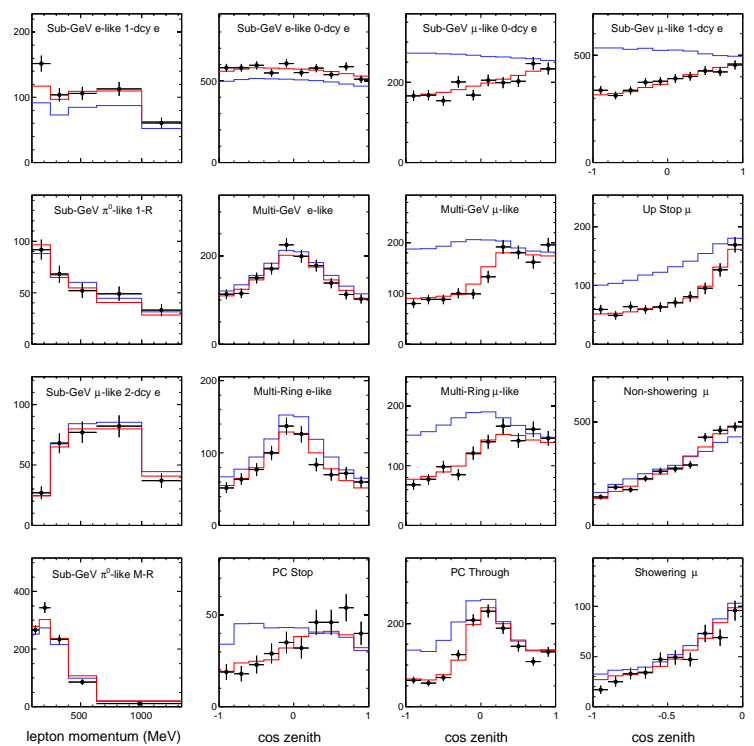

Figure 2: The zenith angle and lepton momentum distributions for each data sample in SK-I+II+III (preliminary). $\cos \Theta=1$ indicates downward-going particles. The blue histograms show the MC prediction without neutrino oscillation and the red histograms show the MC prediction for $v_{\mu} \leftrightarrow v_{\tau}$ oscillations with $\sin ^{2} 2 \theta=1.0$ and $\Delta m^{2}=2.1 \times 10^{-3} \mathrm{eV}^{2}$.

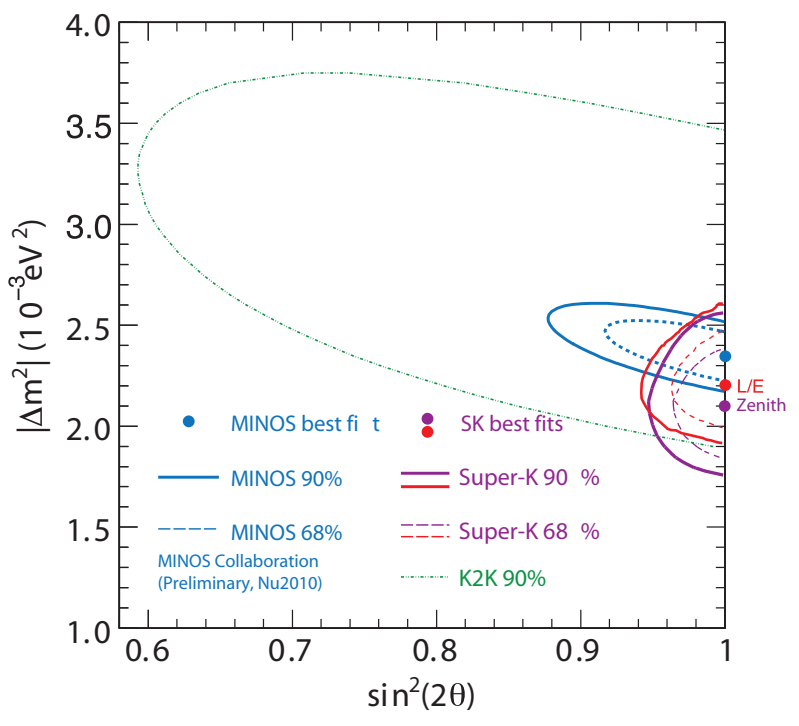

Figure 3: Allowed region of $v_{\mu} \rightarrow v_{\tau}$ neutrino oscillation parameters obtained by SK using contained atmospheric neutrino events and upward-going muon events in SK-I+II+III (preliminary). Solid and dashed contours correspond to 90 and $68 \%$ C.L. respectively. Purple contours are obtained by the zenith angle analysis and red contours are obtained by the L/E analysis. Allowed regions by long-baseline neutrino oscillation experiment $\mathrm{K} 2 \mathrm{~K}$ and MINOS are shown in green and blue contours, respectively. 

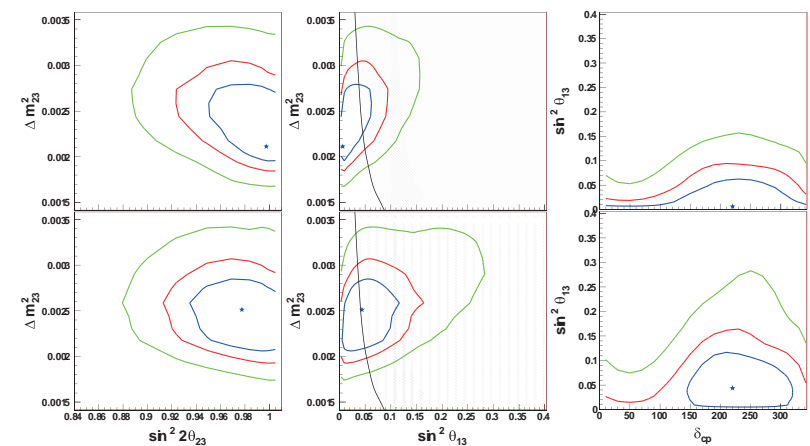

Figure 4: The allowed regions for $\left(\Delta m_{23}^{2}, \sin ^{2} 2 \theta_{23}\right)$; left, $\left(\Delta m_{23}^{2}\right.$, $\left.\sin ^{2} \theta_{13}\right)$; middle, and $\left(\sin ^{2} \theta_{13}, \delta_{c p}\right)$; right for the normal (upper figure) and inverted (lower figure) hierarchy in SK-I+II+III (preliminary). The blue, red, and green contours correspond to 68,90 and $99 \%$ C.L. allowed regions obtained by this analysis, respectively. The shaded regions corresponds to the area excluded at $90 \%$ C.L. by the CHOOZ experiment.

results are summarized in Table 2, All fits are consistent with the two flavor oscillation results and $\mathrm{CHOOZ}$ experiment's upper limit on $\theta_{13}$. No preference for either mass hierarchy exists in the data.

We also performed a test for CPT violations using the atmospheric neutrino data in SK-I+II+III. We applied the two-neutrino disappearance models for neutrinos and anti neutrinos, separately:

$$
\begin{aligned}
& P\left(v_{\mu} \rightarrow v_{\mu}\right)=1-\sin ^{2} 2 \theta \sin ^{2}\left(1.27 \Delta m^{2} L / E\right), \\
& P\left(\bar{v}_{\mu} \rightarrow \bar{v}_{\mu}\right)=1-\sin ^{2} 2 \bar{\theta} \sin ^{2}\left(1.27 \Delta \bar{m}^{2} L / E\right) .
\end{aligned}
$$

The allowed region for anti-neutrino mixing parameters is shown in Figure 5 . The preliminary bestfit parameters are $\left(\Delta m^{2}, \Delta \bar{m}^{2}, \sin ^{2} 2 \theta, \sin ^{2} 2 \bar{\theta}\right)=($ $\left.2.2 \times 10^{-3} \mathrm{eV}^{2}, 2.0 \times 10^{-3} \mathrm{eV}^{2}, 1.0,1.0\right)$. The atmospheric mixing parameters for anti-neutrino oscillations are consistent with those for neutrinos and therefore no evidence for CPT violation is found.

\section{Solar neutrino results}

SK detects solar neutrinos through neutrino-electron elastic scattering (ES), $v+e \rightarrow v+e$, where the energy, direction and time of the recoil electron are measured. Due to its large fiducial mass of $22.5 \mathrm{kton}$, SK gives a precise measurement of the solar neutrino flux, energy spectrum, and time variation via the ES reaction.

In May 2010, we have summarized a preliminary solar neutrino analysis results in SK-III. In SK-III, we have re-estimated all the systematic uncertainties. The preliminary systematic uncertainties on total flux in SKIII are summarized in Table 5 The systematic uncertainty on the total flux is estimated to be $2.1 \%$. This is

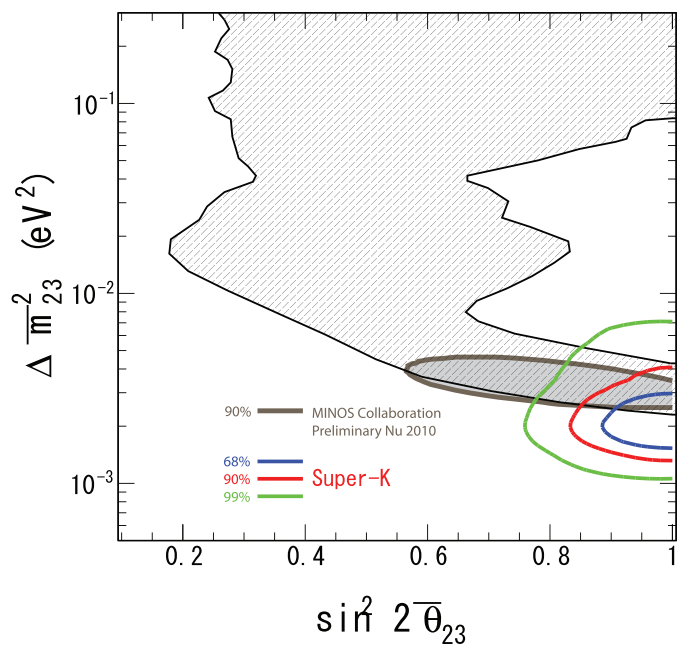

Figure 5: Allowed regions for the anti-neutrino mixing parameters in SK-I+II+III (preliminary). The blue, red and green contours correspond to the $68 \%, 90 \%$, and $99 \%$ C.L. allowed regions, respectively. Shaded region shows the allowed regions by MINOS experiment.

\begin{tabular}{lc}
\hline \hline Source & Total Flux \\
\hline Energy scale & \pm 1.4 \\
Energy resolution & \pm 0.2 \\
${ }^{8}$ B spectrum & \pm 0.2 \\
Trigger efficiency & \pm 0.5 \\
Fiducial volume (vertex shift) & \pm 0.54 \\
Event Reduction & \pm 0.65 \\
Small cluster hits cut & \pm 0.5 \\
Spallation & \pm 0.2 \\
External event cut & \pm 0.25 \\
Background shape & \pm 0.1 \\
Angular resolution & \pm 0.67 \\
Signal extraction method & \pm 0.7 \\
Cross section & \pm 0.5 \\
Live time & \pm 0.1 \\
\hline Total & \pm 2.1 \\
\hline \hline
\end{tabular}

Table 5: Summary of the preliminary systematic uncertainties on total flux in \% in SK-III. 


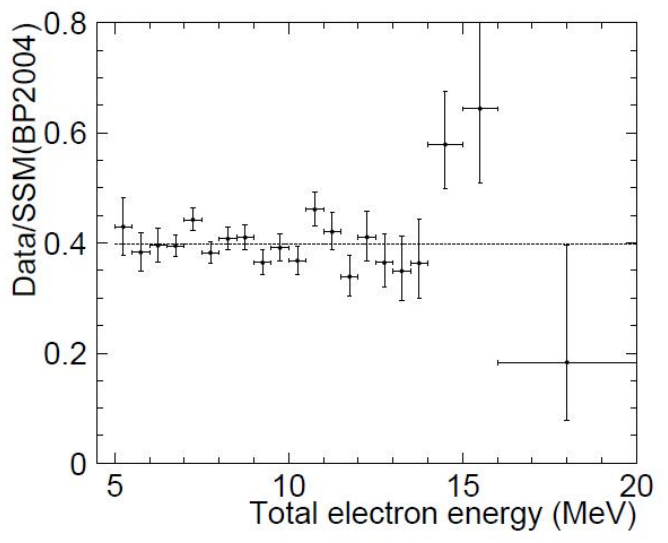

Figure 6: Energy spectrum of solar neutrino flux in SK-III data from 5.0 $\mathrm{MeV}$ to $20 \mathrm{MeV}$ (preliminary). Each point shows the ratio of the data and the expected event rate calculated from the SSM. The line indicates the averaged value of the SK-III data.

about two thirds of the corresponding SK-I value. The main contributions to the improvement are the vertex shift, angular resolution, and event selection uncertainties.

The total live time for solar neutrino analysis in SKIII is 548 days for $E_{\text {total }} \geq 6.5 \mathrm{MeV}$, and 289 days for $E_{\text {total }}<6.5 \mathrm{MeV}$. The recoil electron's total energy range used for this analysis is from 5.0 to $20 \mathrm{MeV}$. The observed ${ }^{8} \mathrm{~B}$ solar neutrino flux via the ES reaction is $2.32 \pm 0.04$ (stat.) \pm 0.05 (syst.) $\left(\times 10^{6} / \mathrm{cm}^{2} / \mathrm{s}\right)$, which is consistent with the previous SK-I and II results. The obtained day-night asymmetry is $\frac{\left(\Phi_{\text {Day }}-\Phi_{\text {Night }}\right)}{\left(\Phi_{\text {Day }}+\Phi_{\text {Night }}\right) / 2}=$ $-0.056 \pm 0.031$ (stat.) \pm 0.013 (syst.). The total ${ }^{8} \mathrm{~B}$ and hep flux values are referred from the BP2004 Standard Solar Model (SSM) [3]. We used the expected spectrum of ${ }^{8} \mathrm{~B}$ and hep neutrinos calculated by Winter [4] and Bahcall [5], respectively.

Figure 6 shows the observed recoil electron's energy spectrum divided by the expected signal rate from BP2004 SSM without oscillation. The line in Figure 6 represents the total SK-III average (flat data/SSM prediction without MSW effect). This result indicates no significant spectral distortion.

A preliminary oscillation analysis has also been done including the SK-III solar neutrino data. The two flavor analysis for the determination of the solar oscillation parameters, $\theta_{12}$ and $\Delta m_{12}^{2}$ was performed with the combined SK-I, II and III data. In this analysis, the total ${ }^{8} \mathrm{~B}$ flux is constrained by the averaged NC flux from SNO NCD [6] and LETA [7] $\left(=(5.14 \pm 0.21) \times 10^{6} / \mathrm{cm}^{2} / \mathrm{s}\right)$. The result is shown in the left side in Figure 7. As

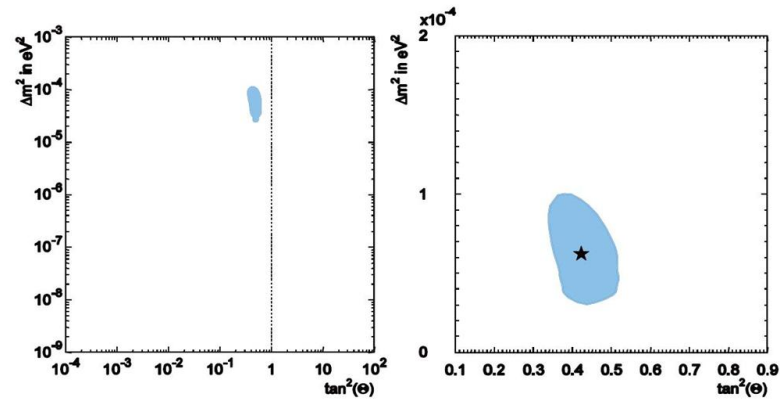

Figure 7: Allowed region (95\% C.L.) for neutrino oscillation parameters, $\Delta m^{2}$ and $\tan ^{2} \theta$ from solar analysis (preliminary). The left figure shows the results from the SK-I, II and III combined analysis with the ${ }^{8} \mathrm{~B}$ flux constrained by SNO NC measurements. The right figure presents the result of global analysis including SK and other solar experiments (SNO, Borexino, Homestake, GALLEX-GNO and SAGE). The star mark indicates the best-fit parameter set.

shown in the left figure, the LOW solution is excluded and the LMA solution is only allowed to explain the solar neutrino oscillation. We have also done a preliminary global oscillation analysis with other solar experiments. In this analysis, the following observation results are used; SNO total CC rates observed in the 306-day pure $\mathrm{D}_{2} \mathrm{O}$ phase (SNO-I) [8], 391-day salt phases (SNOII) [9], and 385-day NCD phase (SNO-III) [6], the combined NC rates of LETA [7] and SNO-III, the predicted day-night asymmetry for SNO-I and II, ${ }^{7} \mathrm{Be}$ solar neutrino flux from Borexino [10], Homestake [11], GALLEX-GNO [12], and SAGE [13]. The result is shown in the right side in Figure 7. The best-fit parameter set is $\tan ^{2} \theta_{12}=0.42_{-0.02}^{+0.04}$ and $\Delta m_{21}^{2}=\left(6.2_{-1.9}^{+1.1}\right) \times$ $10^{-5} \mathrm{eV}^{2}$. In addition, combining the above and KamLAND data [14], the best-fit parameter set becomes $\tan ^{2} \theta_{12}=0.44 \pm 0.03$ and $\Delta m_{21}^{2}=(7.6 \pm 0.2) \times 10^{-5} \mathrm{eV}^{2}$.

We also performed a preliminary 3 -flavor oscillation analysis. In the 3-flavor analysis, the calculation of oscillation probability is based on reference [15]. The probability can be calculated with three parameters: $\theta_{12}$, $\theta_{13}$, and $\Delta m_{12}^{2}$, assuming $\Delta m_{12}^{2} \ll \Delta m_{23}^{2} \sim \Delta m_{13}^{2}$. We fixed $\Delta m_{23}^{2}=2.4 \times 10^{-3} \mathrm{eV}^{2}$ and the normal hierarchy is assumed. For the solar neutrino oscillation, the other mixing parameters are irrelevant, but we set $\theta_{23}=\pi / 4$ and $\delta_{C P}=0$ in our calculation.

As done for the two-flavor analysis, the oscillation probabilities depending on different zenith angles are calculated. Other experiment data are have been published by Homestake, SAGE, Gallex-GNO, Borexino, SNO, and KamLAND. The oscillation parameters are scanned in the following regions: $10^{-5} \mathrm{eV}^{2}<\Delta m_{12}^{2}<$ 


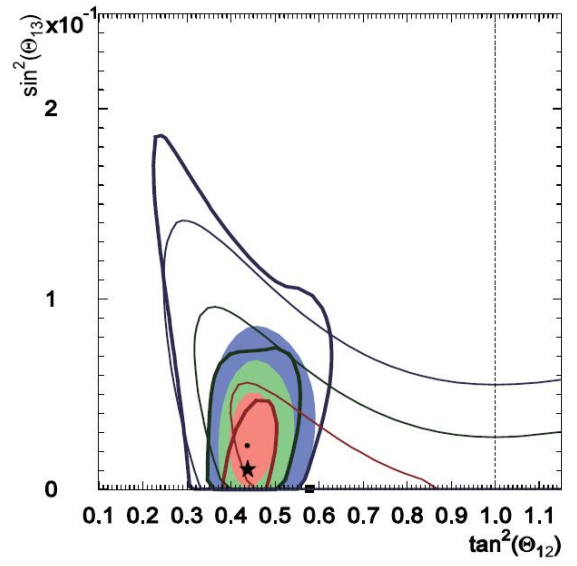

Figure 8: Allowed region for $\sin ^{2} \theta_{13}$ and $\tan ^{2} \theta_{12}$ from the preliminary 3 -flavor analysis. The thick lines and the star mark show the allowed regions and the best-fit point of the solar global analysis. The thin lines and the square mark show the allowed regions and the bestfit point of our KamLAND analysis. The filled areas and the filled circle mark show the allowed regions and the best-fit point of the combined analysis. For all regions, the innermost area (red), the middle area (green) and the outermost area (blue) show 68.3, 95, $99.7 \%$ C.L. respectively.

$2 \times 10^{-4} \mathrm{eV}^{2}, 0.1<\tan ^{2} \theta_{12}<1.0$, and $0<\sin ^{2} \theta_{13}<$ 0.25 .

Figure 8 shows the allowed region for $\sin ^{2} \theta_{13}$ and $\tan ^{2} \theta_{12}$ extracted from this analysis. The allowed region from the combined analysis of solar global and KamLAND analyses is also indicated. The global solar analysis finds that the upper limit of $\sin ^{2} \theta_{13}$ is 0.060 at $95 \%$ C.L. After combining with the KamLAND result, the best fit value of $\sin ^{2} \theta_{13}$ is found to be $0.025_{-0.016}^{+0.018}$ and the upper limit is obtained as $\sin ^{2} \theta_{13}<0.059$ at $95 \%$ C.L.

\section{Conclusion}

Super-Kamiokande-IV is running with the lowest energy threshold in SK. Currently, $100 \%$ efficiency is at $E_{\text {total }}=4.5 \mathrm{MeV}$.

Preliminary results of the full 3-flavor atmospheric neutrino oscillation analysis from SK-I+II+III data are obtained. There is no preference for either mass hierarchy in the data, and no significant constraint applied on CP phase at $90 \%$ C.L.

A CPT violation study on atmospheric neutrino is also done with SK-I+II+III data. No evidence for CPT violation is found, but this is preliminary.

Preliminary results of the solar neutrino measurement in SK-III are obtained. The measured solar ${ }^{8} \mathrm{~B}$ flux via ES reaction is $(2.32 \pm 0.04$ (stat.) \pm 0.05 (sys. $)) \times$ $10^{6} \mathrm{~cm}^{-2} \mathrm{~s}^{-1}$. In a preliminary 3 -flavor global solar neutrino oscillation analysis with KamLAND, the best-fit value of $\sin ^{2} \theta_{13}$ is found to be $0.025_{-0.016}^{+0.018}$ and an upper bound is obtained as $\sin ^{2} \theta_{13}<0.059$ at $95 \%$ C.L.

\section{Acknowledgments}

The author gratefully acknowledges the cooperation of the Kamioka Mining and Smelting Company. SuperKamiokande has been built and operated from funds provided by the Japanese Ministry of Education, Culture, Sports, Science and Technology, the U.S. Department of Energy, and the U.S. National Science Foundation. This work was partially supported by the Research Foundation of Korea (BK21 and KNRC), the Korean Ministry of Science and Technology, the National Science Foundation of China, and the Spanish Ministry of Science and Innovation (Grants FPA2009-13697-C0402 and Consolider-Ingenio-2010/CPAN).

\section{References}

[1] The Super-Kamiokande Collaboration, Nucl. Instr. and Meth. A501 (2003) 418.

[2] S. Yamada et al., IEEE Trans. Nucl. Sci. 57 (2010) 428.

[3] J.N. Bahcall and M.H. Pinsonneault, Phys. Rev. Lett 92 (2004) 121301.

[4] W.T. Winter et al., Phys. Rev. C 73 (2006) 025503.

[5] J.N. Bahcall et al., Nucl. Phys. B (Proc. Suppl.) 77 (1999) 64.

[6] B. Aharmim et al., Phys. Rev. Lett. 101 (2008) 011301.

[7] B. Aharmim et al., Phys. Rev. C 81 (2010) 055504.

[8] B. Aharmim et al., Phys. Rev. C, 75 (2007) 045502.

[9] S.N. Ahmed et al., Phys. Rev. C. 72 (2005) 055502.

[10] C. Arpesella et al., Phys. Rev. Lett. 101 (2008) 091302.

[11] B.T. Cleveland et al., Astrophys. J. 496 (1998) 505.

[12] M. Altmann et al., Phys. Lett. B 490 (2000) 16; also presented at Neutrino 2004 by C.Cattadori.

[13] J.N. Abdurashitov et al., Phys. Rev. C 60 (1999) 055801.

[14] S. Abe et al., Phys. Rev. Lett. 100 (2008) 221803.

[15] V. Barger et al., Phys. Rev. D. 22 (1980) 2718. 